
portantes avancées dans la compréhension des fondements biologiques de notre moralité. Mais la dissociation qui existe souvent entre les actions et les jugements moraux qui les précèdent reste inexplorée. Des indices font penser que le jugement moral serait différent du choix comportemental parce qu'il n'implique pas de conséquences personnelles directes. Les protocoles souvent utilisés qui reposent sur des dilemmes moraux devraient donc être distingués selon que les questions portent sur un jugement objectif sans conséquences : «est-il acceptable de? 》 ou un choix comportemental avec conséquences personnelles potentielles : « accepteriez-vous de ? ». En effet, ce changement de référentiel impliquerait des processus cognitifs différents et pourrait donc constituer un paramètre essentiel de l'étude de notre moralité. <
> Les neurosciences cognitives ont permis d'im-

\section{Imagerie et cognition (9) \\ La nécessité de distinguer \\ le jugement \\ et le choix \\ subjectif dans \\ les neurosciences \\ cognitives de la morale}

Sébastien Tassy

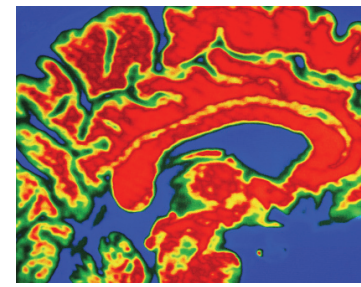

\author{
Pôle universitaire de psychiatrie, \\ Hôpital Sainte-Marguerite, \\ 270, boulevard Sainte-Marguerite, \\ 13009 Marseille, France ; \\ Institut de neurosciences \\ cognitives de la Méditerranée, \\ UMR 6193 CNRS-Université \\ de Provence, Marseille, France. \\ sebastien.tassy@ap-hm.fr
}

probablement neurale entre les processus conduisant à un jugement moral et ceux conduisant au choix comportemental effectif.

\section{Lien entre morale et fonctionnement cérébral}

L'élucidation de la faculté à différencier le bien du mal a alimenté les réflexions de la philosophie, de la psychologie et, depuis quelques années, des neurosciences cognitives. Cette discipline a permis des avancées intéressantes dans la compréhension de la morale. Cependant, il semble qu'elle ignore largement un élément essentiel de la moralité humaine : la connaissance des règles morales ne conditionne pas toujours les comportements qui y ont trait. Nous nous autorisons fréquemment de petits écarts de conduite que notre jugement réprouve, et les transgressions les plus immorales sont bien souvent conscientes. Le lien entre d'une part, notre capacité à déterminer ce qui est bien ou mal, et d'autre part nos choix comportementaux n'est pas univoque. Sa compréhension constitue donc un élément essentiel de l'exploration de la moralité. Cependant, à ce jour, la relation entre nos choix comportementaux et les jugements qui semblent les précéder n'a pas été étudiée par les neurosciences cognitives. Pourtant, il existe des arguments en faveur d'une dissociation cognitive et
Les philosophes puis les psychologues se sont aussi divisés sur la nature de notre sens moral comme processus émotionnel non rationnel ou au contraire cognitif raisonné. S'appuyant sur les travaux de Piaget [1], Kohlberg a proposé un modèle du développement du raisonnement moral en six stades [2] dépendant du développement des capacités de raisonnement de l'enfant. Mais cette vision ignore largement l'importance des émotions dans le processus psychologique aboutissant au jugement moral. À la lumière de la biologie de l'évolution, l'étude des comportements animaux a depuis suggéré que les émotions conduisent les individus à prêter attention au bien-être des autres et à coopérer [3]. Les émotions seraient ainsi à l'origine de la moralité humaine. Dans ce courant émotiviste, Haidt a proposé en 2001 le modèle « intuitionniste social » [4] selon lequel nous voyons une action ou nous entendons une histoire et nous éprouvons alors un sentiment d'approbation ou de désapprobation, une intuition chargée d'affect, dans laquelle le dégoût a un rôle primordial.

Aujourd'hui, les neurosciences cognitives permettent d'approfondir encore l'étude du phénomène moral. Bien que n'ayant pas pour objectif spécifique d'explorer la cognition morale, les neuropsychologues 
ont constaté qu'il existait un lien fort entre l'intégrité de certaines structures cérébrales, en particulier le cortex préfrontal, et des comportements déviant des normes morales. L'exemple de Phinéas Gage devenu socialement inapte après une lésion préfrontale est le plus ancien et le plus illustre [5], mais de nombreux autres cas ont été rapportés, essentiellement chez des patients présentant des lésions préfrontales [6]. En outre, on a pu conclure qu'il existe un lien entre la capacité à générer des émotions sociales, à établir un jugement moral, et à acquérir progressivement des normes sociales complexes, à partir de l'étude de patients adultes ayant acquis des lésions préfrontales durant la première enfance [7].

J.R. Blair, psychiatre anglais, est le premier auteur à s'être véritablement intéressé au lien entre morale et fonctionnement cérébral. II a étudié la capacité de patients psychopathes à distinguer les règles dites «morales » de celles dites « conventionnelles»[8]. Cette distinction avait été proposée par Turiel [9]. Elle repose sur la constatation de la capacité de jeunes enfants à distinguer la transgression de certaines règles (les règles morales) comme étant plus graves et indépendantes de l'existence d'une autorité qui les impose, de la transgression d'autres règles (les règles conventionnelles) apparaissant moins graves et dépendantes de l'existence d'une autorité. Ainsi, par exemple, la transgression d'une règle morale serait « de frapper son voisin »; d'une règle conventionnelle de «manger avec les doigts ». Blair a trouvé que les psychopathes qui transgressent fréquemment et gravement les règles morales, sans montrer d'empathie ou de remord, sont incapables de percevoir la gravité et le caractère indépendant de l'existence d'une autorité d'une transgression morale. Inspiré par K. Lorentz qui a décrit chez les mammifères sociaux un mécanisme d'inhibition d'agression d'un congénère quand celui-ci montre des signes de soumission, Blair a suggéré que chez ces patients, ce mécanisme (qu'il a appelé violence inhibition mechanism, VIM) serait déficient. L'incapacité à distinguer règle morale/conventionnelle viendrait de l'incapacité à ressentir la souffrance d'autrui. Par la suite, Nichols a montré que la distinction règle morale/conventionnelle nécessitait aussi l'apprentissage de repères normatifs abstraits associés à des processus affectifs [10]. Le dysfonctionnement précoce de deux structures neurales particulièrement impliquées dans les processus affectifs, l'amygdale et le cortex préfrontal médian (MPFc), serait en grande partie à l'origine du dysfonctionnement du VIM et donc de la psychopathie [11]. Ces patients ne ressentiraient pas de réponses émotionnelles négatives fortes en percevant la souffrance d'autrui [30] $(\rightarrow)$.

$\rightarrow$ Voir Éditorial de Julie Grèzes, $\mathrm{m} / \mathrm{s} 2011$, $n^{\circ} 8-9$, page 683

\section{Résolution de dilemnes moraux}

Les travaux de J.D. Greene et collaborateurs constituent une autre étape essentielle dans l'identification des processus neurobiologiques qui sous-tendent notre sens moral. Ces chercheurs ont enregistré l'activité neurale en imagerie par résonance magnétique fonctionnelle (IRMf) pendant la résolution de dilemmes moraux, du type « peut-on sacrifier une personne pour en sauver plusieurs autres? » [12]. Ces dilemmes reposent sur des situations dans lesquelles deux impératifs moraux s'opposent, ici ne pas tuer une personne et devoir sauver plusieurs personnes. Suivant les types de conflits moraux rencontrés, le profil d'activation des structures neurales mises en jeu est différent. La résolution d'un dilemme du type : « un tramway dont les freins ont lâché fonce vers un groupe de cinq personnes. Vous vous trouvez sur une passerelle au-dessus de la voie. La seule façon d'empêcher la mort des cinq personnes est de pousser devant le tramway un inconnu de taille imposante qui est sur le pont. Est-il acceptable pour vous de pousser l'inconnu pour sauver les cinq personnes? », qui implique une violence directe et «personnelle », entraîne une activité cérébrale intense dans les structures cérébrales habituellement associées aux émotions, en particulier le cortex préfrontal médian. À l'opposé, dans un contexte de violence plus « impersonnelle » du type : « un tramway dont les freins ont lâché fonce vers un groupe de cinq personnes. La seule chose à faire pour empêcher la mort des cinq personnes est d'aiguiller le chariot sur une autre voie, où il ne tuera qu'une seule personne. Est-il acceptable pour vous de pousser l'aiguillage pour sauver les cinq personnes? », les structures associées au raisonnement et au contrôle cognitif, en particulier le cortex préfrontal latéral ( $L P F c)$, sont intensément activées. D'autres études ont depuis retrouvé des profils d'activations cérébrales qui impliquent clairement à la fois les réseaux des affects et ceux du raisonnement et du contrôle cognitif dans des situations de jugements moraux [13, 14]. À l'opposé d'une conception unitaire des fondements rationnels ou émotionnels de notre moralité, ces résultats soutiennent la vision d'un système alternatif, dynamique, dans lequel des processus affectifs automatiques et rationnels contrôlés s'opposent pour générer un jugement/ décision moral(e) dépendant(e) du contexte.

\section{Le modèle dual de J.D. Greene : compétition entre affectif et cognitif}

Ce que J.D. Greene nomme «le jugement moral » repose sur un système dual de traitement de l'information dans lequel les structures cognitives, en particulier le LPFc, contrôlent en les inhibant les réponses émotionnelles dépendantes de structures comme le MPFc. L'équilibre entre les structures cognitives qui assurent un rôle de contrôle rationnel et les structures générant des réactions émotionnelles instinctives conditionnerait «le jugement moral ». La perturbation de l'une des deux composantes de ce système conduirait donc à l'action prédominante de l'autre.

Lors de la résolution de dilemmes moraux, réduire la réaction émotionnelle instinctive conduirait à une prédominance du contrôle rationnel, conduisant à favoriser 
une solution plus utilitariste (rationnelle), celle qui permet de sauver le plus grand nombre de vies. Pour tester cette hypothèse, Damasio et son équipe ont demandé à des patients ayant un déficit acquis des réactions émotionnelles résultant de lésions situées dans le MPFc, de résoudre les dilemmes moraux. Conformément aux anticipations faites à partir de ce modèle, ces patients ayant une lésion cérébrale se sont montrés «hyper-rationnels » dans certains de leurs jugements moraux [15]. Faisant preuve de moins d'états d'âme, ils ont, plus fréquemment que les sujets sains, accepté l'idée de sacrifier un individu si cela pouvait permettre d'en sauver plusieurs.

Pourtant, en totale contradiction avec les prévisions du modèle dual, ces mêmes patients se sont montrés « hyper-émotionnels », irascibles et irrationnels dans leurs réactions face à un autre type de dilemme social, celui posé par le jeu de l'ultimatum (UG) [16]. Dans ce jeu destiné à explorer la sensibilité à l'iniquité, ils ont été plus enclins à refuser les offres inéquitables mais avantageuses qui leur étaient faites. Or ce type de refus est habituellement associé à des émotions intenses, en particulier de colère [17].

Ce même jeu a été utilisé pour tester l'autre versant du model dual, en perturbant (transitoirement) le contrôle cognitif du sujet par une stimulation magnétique transcrânienne répétitive sur le LPFc. Encore une fois en totale contradiction avec les prévisions du modèle dual, la suppression du contrôle cognitif n'a pas conduit à l'hyper-expression d'une réaction émotionnelle impulsive. Les sujets dont le contrôle cognitif avait été perturbé ont « hyper-rationnellement » accepté les offres inéquitables mais avantageuses qui leur étaient faites [18]. La colère face à l'iniquité n'a plus guidé leurs choix. Ces deux expériences ont donc sérieusement remis en question le modèle dual de Greene et ont apporté du relief au modèle proposé par J. Moll qui synthétise des travaux dépassant le cadre de la cognition morale [14] ${ }^{1}$.

\section{Le modèle de J. Moll : conflit entre deux systèmes} cognitivo-émotionnels différents

Selon J. Moll, le cortex préfrontal permettrait une représentation d'un événement vécu en fonction des séquences qui le composent (appuyé sur l'aiguillage, déviation du train, mort probable de l'ouvrier) et du contexte, en intégrant les informations de nature différente. Ces informations regroupent des indices perceptifs à valence sociale (contact physique, regard du sacrifié) et des connaissances sociales décontextualisées telles que les normes sociétales et morales. Cette intégration dans les cortex frontaux conduirait à une activation du système des affects dans les structures limbiques et paralimbiques. Cet ensemble représenterait un système articulé, appelé event-feature-emotion

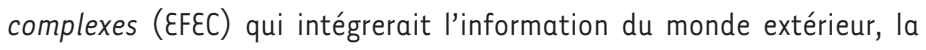
reliant éventuellement à une représentation à contenu « moral » et induisant une réponse comportementale. Dans ce modèle, l'alternative que représente le sacrifice d'un individu pour en sauver cinq (calcul rationnel) et le dégoût de tuer (réaction émotionnelle), conduisent à

${ }^{1}$ Nos résultats publiés très récemment montrent qu'une inhibition du LPFc droit lors de la résolution de dilemmes moraux conduit à des jugements moins émotionnels, contrairement aux prévisions du modèle dual de Greene. Par ailleurs, nous avons retrouvé qu'il existait un effet différent de cette perturbation sur le choix de l'action [31]. un conflit entre deux systèmes cognitivo-émotionnels différents, plutôt qu'à une compétition entre un système cognitif et un autre affectif. II y aurait une dissociation des affects moraux, reposant sur des réseaux corticaux différents [19]. Ainsi, le MPFc participerait davantage aux affects prosociaux tels que la culpabilité, la compassion ou l'attachement, et le LPFc aux affects aversifs plus égocentrés (colère, frustration, dégoût). Cette hypothèse permet d'expliquer que lors de la résolution des dilemmes moraux, les patients avec des lésions du MPFc, privés de la capacité de ressentir de la compassion, acceptent de sacrifier un individu pour en sauver d'autres plus nombreux, mais ils pourraient encore ressentir de la colère face aux offres injustes du jeu de l'ultimatum.

\section{Résolution de dilemmes moraux par les psychopathes}

Dans les hypothèses de Greene et de Moll, les émotions sont au centre de la prise de décision dans un contexte moral. Cela vient renforcer l'hypothèse de Blair d'un déficit émotionnel dans la psychopathie reposant sur un défaut de fonctionnement d'un réseau qui implique le cortex préfrontal médian et l'amygdale. Ce défaut expliquerait leur impossibilité à se conformer à certaines normes morales. Selon le modèle dual de Greene, les psychopathes devraient présenter une diminution des réactions émotionnelles et répondre avec un biais rationnel, donc utilitariste (sauver le plus grand nombre), dans la résolution de dilemmes moraux. Selon le modèle de Moll, un dysfonctionnement du MPFc qui génère des émotions spécifiquement prosociales devrait conduire à une réduction de la compassion pour la victime dans les situations de dilemmes et conduire aussi à un biais utilitariste. Cependant, lors de la résolution de dilemmes moraux, les patients psychopathes ont répondu de manière identique aux sujets sains, ils n'ont pas montré ce biais utilitariste qu'on aurait pu attendre $[20,21]$. Les prédictions des deux modèles concernant les jugements moraux élaborés par les psychopathes ne sont pas bonnes, ce qui les invalide partiellement. Ainsi, les patients psychopathes jugeraient inacceptable de violenter autrui pour assouvir un besoin. Mais les comportements qui les caractérisent indiquent qu'ils choisissent souvent la violence pour arriver à leurs fins. Cela confirme que comportements et jugements moraux pourraient être dissociés comme cela avait été envisagé dans le cas de patients présentant des lésions préfrontales [6] et comme nous le rappellent tous les jours nos petites transgressions. Les comportements qui traduisent les choix moraux sont donc relativement indépendants du jugement moral. Les processus neuraux mis en jeu lors du jugement et ceux mis en jeu lors du choix moral pourraient donc aussi être dissociés. 


\section{Exemple de dissociation entre jugement perceptif et action}

Il est intéressant de noter qu'une telle dissociation entre un comportement et le jugement évaluatif qui le précède pourrait se rapprocher de certaines dissociations rapportées dans la littérature neuropsychologique. Grâce à une telle dissociation, la voie visuelle dorsale guidant une action orientée automatique a pu être différenciée de la voie visuelle ventrale permettant un jugement perceptif [22]. Un patient ayant une atteinte de la voie visuelle ventrale souffre d'agnosie visuelle de la forme et est incapable de dire en voyant un rond s'il est différent d'un carré. Cependant, il est capable de saisir les deux objets en ajustant la forme de sa main spécifiquement à chaque objet avant même de l'avoir touché. Le patient n'a donc pas la possibilité d'élaborer un jugement perceptif adapté se rapportant à la forme d'un objet, mais est capable d'avoir un comportement adapté à la forme spécifique de l'objet [23]. La dissociation inverse est retrouvée dans le cas d'une atteinte de la voie visuelle dorsale, le patient souffre alors d'une ataxie optique : il est capable de différencier un rond d'un carré, mais il est incapable d'ajuster la forme de sa main pour saisir le rond différemment du carré [24]. Une action adaptée ne requiert donc pas forcément un jugement perceptif normal, et à l'inverse, un jugement perceptif normal ne conduit pas forcément à une action adaptée. Les psychopathes pourraient donc être l'exemple de patients présentant une capacité de jugement moral perceptif préservée mais des choix comporte-

\section{GLOSSAIRE}

Cortex préfrontal : la partie antérieure du lobe frontal du cerveau, située en avant des régions motrices et prémotrices. Cette région est le siège de différentes fonctions cognitives dites supérieures.

Cortex préfrontal médian (MPFc) : partie du cortex préfrontal située sur la face externe des lobes frontaux (la partie visible de côté).

Cortex préfrontal latéral ( $L P F c)$ : partie du cortex préfrontal située sur la face interne des lobes frontaux (entre les hémisphères cérébraux).

Structures limbiques : nom donné à un groupe de structures du cerveau jouant un rôle très important dans le comportement, et en particulier dans diverses émotions comme l'agressivité, la peur, le plaisir ainsi que la formation de la mémoire. Ces structures sont situées dans la profondeur du cerveau.

Psychopathe : individu présentant un trouble de la personnalité (appartenant au groupe personnalité antisociale) se traduisant notamment par : une incapacité à se conformer à des normes sociales, des comportements délinquants ; le mensonge répété ; l'impulsivité ; l'agressivité ; le mépris pour sa sécurité ou celle d'autrui ; l'irresponsabilité et l'absence de remord.

Jeu de l'ultimatum (ultimatum game - UG) : jeu d'économie expérimentale dans lequel un joueur $A$ reçoit une somme qu'il doit partager avec un joueur $B$, selon la règle du jeu suivante : si $B$ accepte le partage, $A$ et $B$ repartent chacun avec la somme correspondante; si, en revanche $B$ refuse l'offre, A perd tout et $A$ et $B$ repartent sans rien.

II a très largement été montré (et cela pour différentes cultures et différentes sommes) que si A donne moins de $25 \%$ - $30 \%$ de ce qu'il reçoit à $B, B$ refuse. Ce refus contredit la théorie du choix rationnel qui voudrait que $B$ accepte n'importe quelle somme, celle-ci valant toujours mieux que de tout perdre. mentaux inadaptés du point de vue moral. À ce jour, cette hypothèse n'a jamais était étudiée, mais il existe des pistes de réflexions.

\section{Intervention des conséquences personnelles} dans la différence jugement/choix

Une des distinctions essentielles de ce que nous avons appelé le jugement et le choix est que le choix implique des conséquences personnelles qui n'existent pas pour le jugement [25]. Cela se rapproche d'une distinction fondée sur une différence de perspective face à une situation présentée. Pour résoudre un choix, le sujet doit être acteur réel ou simulé (se projetant à la place des protagonistes), il se place dans un référentiel égocentré et adopte un mode de résolution subjectif. Au contraire, le jugement repose sur un mode d'évaluation qui n'implique pas de conséquence pour le sujet lui-même. II ne se projette pas et reste dans une évaluation objective à un niveau très abstrait. Il est dans un référentiel que Frith et de Vignemont disent allocentré [26, 27]. Une telle différence de référentiel d'évaluation serait une dimension essentielle pour appréhender la cognition sociale. Dans le cadre des dilemmes moraux, il a été montré que les éventuelles conséquences personnelles induites par un changement de perspective sont un facteur déterminant dans les réponses $[28,29]$. Cette distinction peut être clairement mise en œuvre en modulant les conséquences possibles par une légère variation dans la façon dont le dilemme est présenté. Une question du type «sacrifieriez-vous une personne pour en sauver plusieurs ? » implique des conséquences personnelles (simulées), elle se rapproche donc d'un choix comportemental. Au contraire, la question « est-il acceptable de sacrifier une personne pour en sauver plusieurs? » n'implique aucune conséquence personnelle et se rapproche d'un jugement. Pourtant, à ce jour, les expérimentations menées avec ces dilemmes n'ont pas tenu compte de cette subtilité dans la façon de présenter les questions et ce qui est appelé « jugement moral » est indifféremment une réponse à une question induisant un jugement ou un choix comportemental. Bien que la différence jugement/choix paraisse être un paramètre essentiel dans l'étude de la cognition morale, elle reste largement ignorée des paradigmes expérimentaux des neurosciences cognitives. S'appuyer sur cette distinction permettra d'approfondir la compréhension des bases neurales des phénomènes moraux. $\diamond$

\section{SUMMARY}

On the necessity to distinguishing judgment from subjective choice in the cognitive neuroscience of morality Recently, cognitive neuroscience has shed new light on our understanding of the neural underpinning of 
humans' morality. These findings allow for a fundamental questioning and rethinking of the alleged dichotomy between reason and emotion, that has profoundly shaped both moral philosophy and moral psychology. Functional neuroimaging and neuropsychology studies have provided strong arguments favoring a dynamic and interdependent interaction between rational and emotional processes in the brain. Yet another fundamental issue remains largely unexplored: the dissociation between certain behaviours and the moral judgments that seem to precede them. The importance of this dissociation was highlighted in a study of psychopathic patients during which they preserved their moral judgments while frequently engaging in completely non moral behaviour. Such dissociation could result from the cognitive difference between an objective moral judgement with no personal consequence, and a subjective behavioural choice that has effective or potential personal consequences. Consequently, the results of moral dilemma experiments would differ widely depending whether they explore objective or subjective moral evaluations. That these evaluations involve two distinct neural processes should be taken into account when exploring the neural bases of human morality. $\diamond$

\section{CONFLIT D'INTÉRÊTS}

L'auteur déclare n'avoir aucun conflit d'intérêts concernant les données publiées dans cet article.

\section{RÉFÉRENCES}

1. Piaget J. Le jugement moral chez l'enfant. Paris : F. Alcan, 1932.

2. Kohlberg L. Stage and sequence: the cognitive-developmental approach to socialization. In : Goslin DA, ed. Handbook of socialization theory and research. Boston : Houghton-Mifflin, 1969 : 347-480.

3. De Waal FB. Putting the altruism back into altruism: the evolution of empathy. Ann Rev Psychol $2008 ; 59: 279-300$.

4. Haidt J. The emotional dog and its rational tail: a social intuitionist approach to moral judgment. Psychol Rev $2001 ; 108: 814-34$.

5. Damasio AR. Descarte's error: emotion, reason, and the human brain. New York: Grosset/Putnam, 1994.

6. Eslinger PJ, Damasio AR. Severe disturbance of higher cognition after bilateral frontal lobe ablation: patient EVR. Neurology 1985 ; 35 : 1731-41.

7. Anderson SW, Bechara A, Damasio H, et al. Impairment of social and moral behavior related to early damage in human prefrontal cortex. Nat Neurosci $1999 ; 2$ : 1032-7.

8. Blair R. A cognitive developmental approach to morality: investigating the psychopath. Cognition $1995 ; 57: 1-29$.

9. Turiel $\varepsilon$. The development of social knowledge: morality and convention. Cambridge : Cambridge University Press, 1983.

10. Nichols S. Norms with feeling: towards a psychological account of moral judgment. Cognition $2002 ; 84: 221-36$
11. Blair RJ. The amygdala and ventromedial prefrontal cortex: functional contributions and dysfunction in psychopathy. Philos Trans $R$ Soc Lond B Biol Sci $2008 ; 363: 2557-65$.

12. Greene JD, Sommerville RB, Nystrom LE, et al. An fMRI investigation of emotional engagement in moral Judgment. Science $2001 ; 293: 2105-8$.

13. Greene JD, Nystrom $L \varepsilon$, Engell AD, et al. The neural bases of cognitive conflict and control in moral judgment. Neuron $2004 ; 44: 389-400$.

14. Moll J, Zahn R, de Oliveira-Souza R, et al. Opinion: the neural basis of human moral cognition. Nat Rev Neurosci $2005 ; 6$ : 799-809.

15. Koenigs M, Young L, Adolphs R, et al. Damage to the prefrontal cortex increases utilitarian moral judgements. Nature 2007 ; 446 : 908-11.

16. Koenigs M, Tranel D. Irrational economic decision-making after ventromedial prefrontal damage: evidence from the ultimatum game. J Neurosci 2007 ; 27 : 951-6.

17. Van 't Wout M, Kahn RS, Sanfey AG, Aleman A. Affective state and decisionmaking in the ultimatum game. Exp Brain Res 2006 ; 169 : 564-8.

18. Knoch D, Pascual-Leone A, Meyer K, et al. Diminishing reciprocal fairness by disrupting the right prefrontal cortex. Science 2006 ; 314 : 829-32.

19. Moll J, de Oliveira-Souza R. Moral judgments, emotions and the utilitarian brain. Trends Cogn Sci 2007 ; 11 : 319-21.

20. Tassy S, Oullier 0, Cermolacce M, et al. Do psychopathic patients use their DLPFC when making decisions in moral dilemmas? Mol Psychiatry 2009; 14 : 908-9.

21. Cima M, Tonnaer F, Hauser MD. Psychopaths know right from wrong but don't care. Soc Cogn Affect Neurosci 2010 ; 5 : 59-67.

22. Goodale MA, Milner AD. Separate visual pathways for perception and action. Trends Neurosci 1992; $15: 20-5$

23. Goodale MA, Milner AD, Jakobson LS, Carey DP. A neurological dissociation between perceiving objects and grasping them. Nature 1991 ; 349 : 154-6.

24. Perenin MT, Vighetto A. Optic ataxia: a specific disruption in visuomotor mechanisms. Brain 1988; 111 : 643-74.

25. Sood S, Forehand M. On self-referencing differences in judgment and choice. Organ Behav Hum Decis Proc 2005 ; 98 : 144-54.

26. Frith $U$, deVignemont F. Egocentrism, allocentrism and Asperger syndrome. Conscious Cogn $2005 ; 14: 719-38$.

27. De Vignemont F. Frames of reference in social cognition. $\varrho J$ Exp Psychol $2008 ; 61: 90-100$.

28. Moore AB, Clark BA, Kane MJ. Who shalt not kill? Individual differences in working memory capacity, executive control, and moral judgment. Psychol Sci $2008 ; 19: 549-57$.

29. Nadelhoffer T, Feltz $A$. The actor-observer bias and moral intuitions: adding fuel to Sinnott-Armstrong's fire. Neuroethics 2008 ; 1 : 133-44.

30. Grèzes J. Les émotions, modes d'action et de communication. Med Sci (Paris) $2011 ; 27: 683-4$.

31. Tassy $S$, Oullier 0 , Duclos $Y$, et al. Disrupting the right prefrontal cortex alters moral judgement. Soc Cogn Affect Neurosci $2011 ; 22$ avril (online).
TIRÉS À PART

S. Tassy

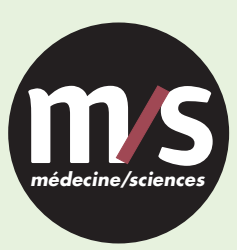

Tarifs d'abonnement $\mathrm{m} / \mathrm{s}-2011$

Abonnez-vous

à médecine/sciences
$>$ Grâce à $m / s$, vivez en direct les progrès des sciences biologiques et médicales

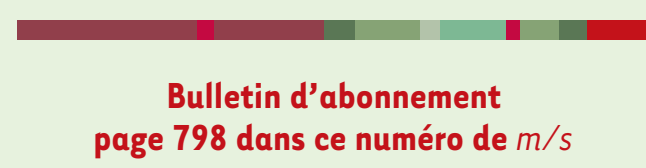

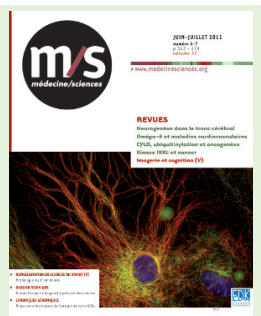

